\section{read2Marker: a data processing tool for microsatellite marker development from a large data set}

\begin{abstract}
Hiroyuki Fukuoka¹, Tsukasa Nunome , Yasuhiro Minamiyama ${ }^{2}$, Izumi Kono ${ }^{3}$, Nobukazu Namiki ${ }^{3}$, and Akio Kojima ${ }^{1}$

${ }^{1}$ National Institute of Vegetable and Tea Science (NIVTS), Ano, Mie, ${ }^{2}$ Kyoto Prefectural Institute of Agricultural Biotechnology, Seika, Kyoto, and ${ }^{3}$ Society for TechnoInnovation of Agriculture, Forestry and Fisheries (STAFF), Tsukuba, Ibaraki, Japan
\end{abstract}

BioTechniques 39:472-476 (October 2005)

doi $10.2144 / 000112025$
Microsatellites, or simple sequence repeats (SSRs), are widely considered to be one of the best sources of DNA polymorphism that can be utilized for developing genetic markers (1). Recently, several methods for making microsatellite-enriched genomic libraries were reported $(2,3)$ and, as a consequence, the random sequencing of a microsatellite-enriched genomic library becomes a possible strategy for the mass development of microsatellite markers. Despite the progress in microsatellite isolation, it remains timeconsuming and laborious to handle the large amounts of sequence data. A set of script programs, read2Marker, was developed as a solution to this problem (Figure 1). In cooperation with phred/ phrap (4), BLAST (5), Primer3 (6), and T-Coffee (7), read2Marker can process an unlimited number of raw output data files from an automatic sequencer. It can run on a MacOS X or Linux platform and generates a report on many aspects of the nonredundant microsatellite-containing clones such as whole sequence, microsatellite core sequences, and PCR primers.

A set of trace data obtained by sequencing from both ends of a clone is base-called by phred and assembled by phrap. Based on quality values (QVs), the high QV part of the assembled sequence is used for further processing. When data in plain text format without QVs are to be processed, all of the nucleotides are automatically assigned a QV of 20 to disable the quality threshold aspect of the read2Marker program. Subsequently, a microsatellite core is screened in the clone sequence by a newly developed tool, srchssr2. Initially, srchssr2 searches for repeated sequences of four dinucleotide motifs (GA, GT, AT, GC) and 10 trinucleotide motifs (AAC, AAG, ACC, ACG, ACT, AGC, AGG, ATC, TAT, CGC) independently in the clone sequence, and the core with the highest repeat number is selected. Next, a core structure neighboring the selected longest core is rescreened. If the gap between these cores is shorter than a maximum gap length set by the user in advance, then the two cores and gap sequence are connected to make a new core sequence. These procedures are repeated, and finally the composite microsatellite core in the sequence is identified. Finally, a PCR primer set for amplifying the microsatellite core is designed using the Primer3 program. The algorithm for finding microsatellites with srchssr2 is quite simple, but it is efficient enough to find cores. It can find composite repeats composed of different motifs and extracts $5^{\prime}$ - and 3 -flanking sequences that will be used for a redundancy check in the next step. Several SSR-finding programs with more sophisticated algorithms such as Msatfinder (M.I. Thurston and D. Field, www.bioinf.ceh.ac.uk/msatfinder), TROLL (8), Sputnik (C. Abajian, espressosoftware.com/pages/sputnik. jsp), and Tandem repeats finder (9) are available, and some of them could be substituted for srchssr 2 with some modification, especially in cases where screening for motifs of more than three nucleotides is required.

In order to remove redundant sequences, a homology check is performed using the BLAST algorithm. Two outer sequences of the clone flanking the microsatellite core are independently set as query sequences and BLASTed to a database comprising unique clones. If at least one of the two core-flanking sequences does not provide a hit from the database, the clone is judged as unique and added to

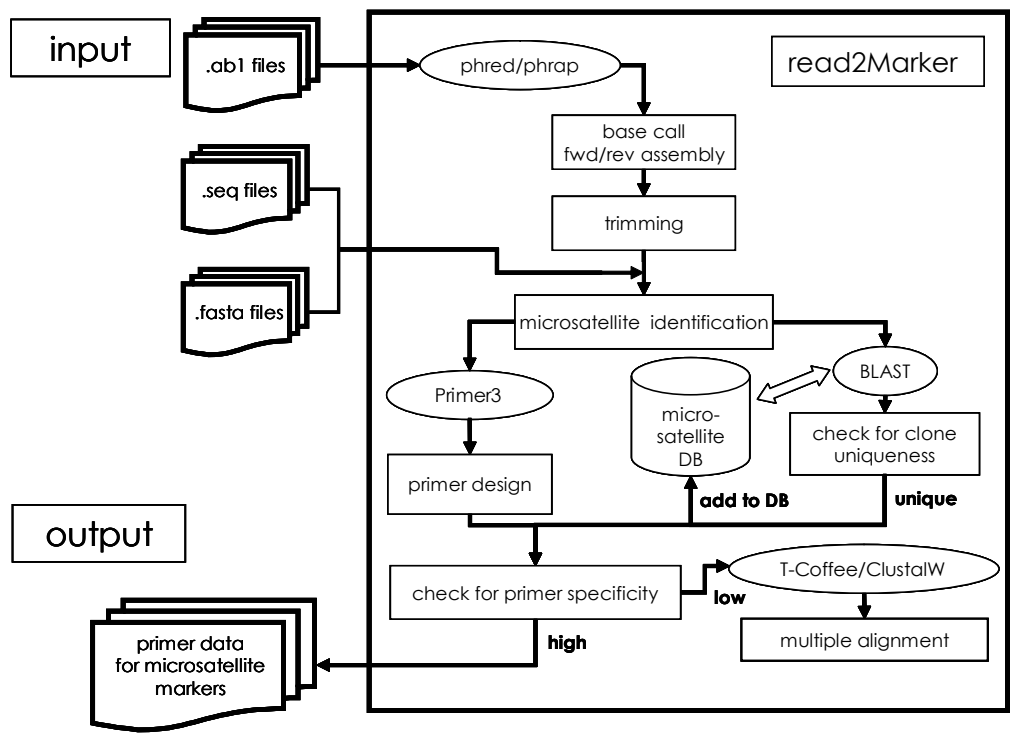

Figure 1. Summary of read2Marker. The box illustrates the steps that are included in read2Marker and processed automatically. Additional programs called out by read2Marker are illustrated as ellipses. fwd/rev, forward/reverse; DB, database. 


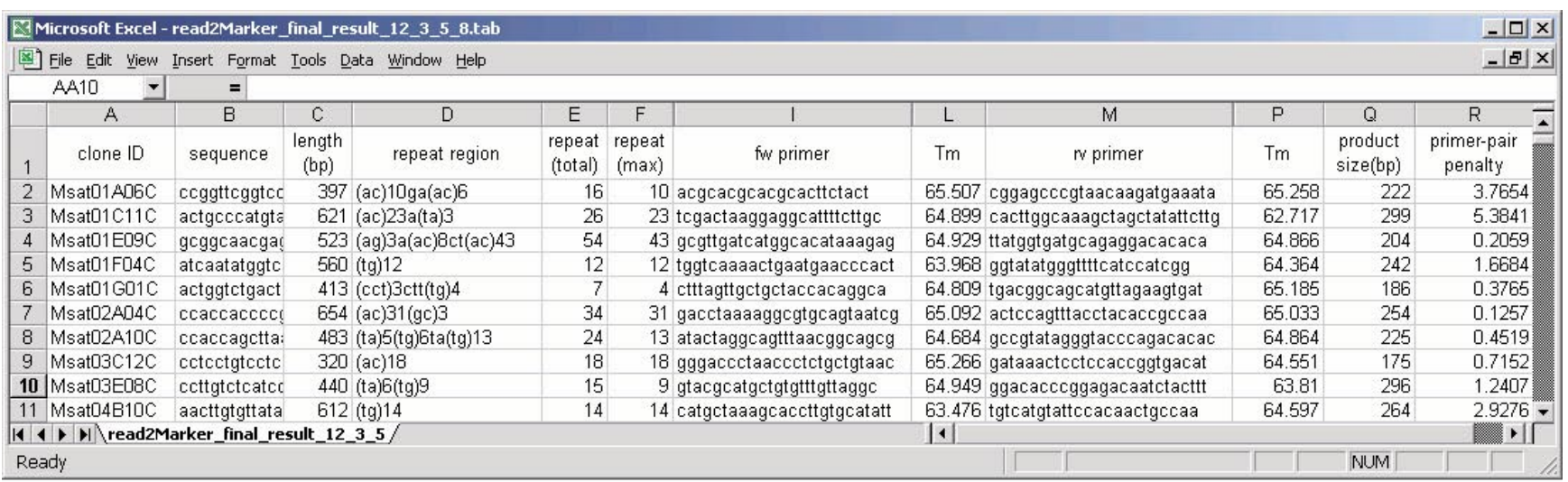

Figure 2. Tab-delimited final result file generated by read2Marker. The file is opened by a spreadsheet program. In the figure, some columns that contain optional information such as primer position and primer length are hidden. fw, forward; rv, reverse; Tm, melting temperature.

the database. However, at the end of this process, some sequences in the database will not be unique but rather are present as duplicates. This situation arises as a consequence of the methodology used to add sequences to the database. Consider two clones, A and B, that are derived from the same microsatellite locus that have overlapping, but not identical, flanking sequences because of incomplete restriction enzyme digestion or some other experimental variable. In this instance, assume also that the $3^{\prime}$-flanking sequence of clone $\mathrm{A}$ is considerably shorter than that of clone B. If we carry out a BLAST analysis using the 3 '-flanking sequence of clone $\mathrm{B}$, then a low score would be given despite clone $\mathrm{A}$ being in the database. Clone B would be assumed to be unique and added to the database, although in reality it is a duplicate. To remove this kind of duplication, all of the sequences in the database are then used in BLAST searches of the database itself. This second BLAST search is effective for the removal of any remaining redundancy in the database.

Occasionally, primer sets designed for different unique sequences share considerable sequence homology because of the presence of common (nonrepeated) sequences adjacent to the microsatellite core and/or the existence of other sequence(s) that share relatively high homology. Therefore, all primer sets should be examined for the possibility of mispriming and subsequent amplification of sequences other than the expected target. All combinations of primer sets and unique clone sequences in the database are examined, and possible mispriming combinations are grouped. The unique clones gathered in a group are aligned by T-Coffee for later use in the manual design of clone-specific primers. After removal of mispriming primer sets, the final result of marker design is provided as an output of tab-delimited text as shown in Figure 2. The file contains clone names, whole clone sequences, microsatellite core sequences, forward and reverse primer sequences, and several other features of the primers such as length, melting temperature $\left(\mathrm{T}_{\mathrm{m}}\right)$, and penalty value assigned by Primer3.

The efficiency of the read2Marker system was evaluated using sequence data obtained from 3456 clones randomly picked from an enriched genomic library of the eggplant, Solanum melongena. In total, 576 primer pairs were designed, and 380 primer sets of the 576 exhibited a single, clear band upon electrophoresis of PCR products. The efficiency of identifying successful primer sets using the read2Marker system (380/576 = $65 \%$ ) compares favorably with our previous manual experiments that gave a rate of $72 \%$. The principal difference lies in the fact that the processing of approximately 500 clones manually took almost a half-month, whereas the analysis of 3456 clones was completed within $6 \mathrm{~h}$ using the read2Marker system. In a similar fashion, random sequence data obtained from approximately 1900 clones in the pepper (Capsicum annuum L.) were processed for the identification of unique clones by read2Marker and also manually with the help of a commercial DNA analyzing program package, GENETYX $^{\circledR}$ (Genetyx, Tokyo, Japan). Of the clones deemed unique by read2Marker, more than $95 \%$ were consistent with those selected manually. These trials confirm that the newly developed system for microsatellite marker development, read2Marker, has a high performance in data processing and overcomes one of the most timeconsuming and laborious steps of microsatellite marker development. The complete set of scripts and instructions are available on request from the corresponding author.

\section{ACKNOWLEDGMENTS}

This work was supported by research program "Development of DNA Marker-Aided Selection Technology for Plants and Animals" (DM-2105) funded by the Ministry of Agriculture, Forestry and Fisheries of Japan.

\section{COMPETING INTERESTS STATEMENT}

The authors declare no competing interests.

\section{REFERENCES}

1.Tautz, D. 1989. Hypervariability of simple sequences as a general source for polymorphic DNA markers. Nucleic Acids Res. 17:64636471.

2.Hamilton, M.B., E.L. Pincus, A. DiFiore, and R. Fleischer. 1999. Universal linker and 


\section{BENCHMARKS}

ligation procedures for construction of genomic DNA libraries enriched for microsatellites. BioTechniques 27:500-507.

3.Zane, L., L. Bargelloni, and T. Patarnello. 2002. Strategies for microsatellite isolation: a review. Mol. Ecol. 11:1-16.

4.Ewing, B., L. Hillier, M. Wendl, and P. Green. 1998. Basecalling of automated sequencer traces using phred. I. Accuracy assessment. Genome Res. 8:175-185.

5.Altschul, S.F., W. Gish, W. Miller, E.W. Myers, and D.J. Lipman. 1990. Basic local alignment search tool. J. Mol. Biol. 215:403410 .

6.Rozen, S. and H. Skaletsky. 2000. Primer3 on the WWW for general users and for biologist programmers, p. 365-386. In S. Krawetz and S. Misener (Eds.), Bioinformatics Methods and Protocols: Methods in Molecular Biology. Humana Press, Totowa, NJ.

7.Notredame, C., D. Higgins, and J. Heringa. 2000. T-Coffee: A novel method for multiple sequence alignments. J. Mol. Biol. 302:205217.

8.Castelo, A.T., W. Martins, and G.R. Gao. 2002. TROLL-tandem repeat occurrence locator. Bioinformatics 18:634-636.

9.Benson, G. 1999. Tandem repeats finder: a program to analyze DNA sequences. Nucleic Acids Res. 27:573-580.

Received 26 June 2005; accepted 26 July 2005.

Address correspondence to Hiroyuki Fukuoka, National Institute of Vegetable and Tea Science (NIVTS), Kusawa 360, Ano, Mie 514-2392, Japan. e-mail: h.fukuoka@affrc.go.jp

To purchase reprints

of this article, contact

Reprints@BioTechniques.com 